\title{
Comment la bibliothèque du musée de l'Homme Yvonne-Oddon rend-elle hommage à Cro-Magnon ?
}

\author{
How the Yvonne-Oddon Library (Musée de l'Homme) Pays Tribute to Cro-Magnon?
}

\author{
N. Charrier-Arrighi $\cdot$ E. Edy $\cdot$ E. Fileyssant \\ Reçu le 5 juillet 2018 ; accepté le 28 août 2018 \\ C) Société d'Anthropologie de Paris et Lavoisier SAS 2018
}

Installée au quatrième étage du musée de l'Homme, la bibliothèque Yvonne-Oddon ${ }^{1}$ a souhaité rendre hommage à Cro-Magnon en célébrant les 150 ans de la découverte du site du même nom par une exposition intitulée «CroMagnon : $150^{\mathrm{e}}$ anniversaire d'une découverte (pré)historique », présentée du 6 juin au 30 novembre 2018.

\section{Premières publications sur la découverte de Cro-Magnon}

Bibliothèque de référence en France pour la préhistoire et l'anthropologie biologique depuis $1983^{2}$, la bibliothèque du musée de l'Homme conserve dans son fonds très riche des documents qui témoignent de l'histoire de ces disciplines. Ainsi y trouve-t-on l'ensemble des premières publications documentant le site de Cro-Magnon.

Dès le printemps 1868, la grotte de Cro-Magnon apparaît dans des articles de revues scientifiques [1-6] puis dans des ouvrages [7-8]. Si une dizaine d'articles est parue en quelques semaines sur la découverte et le site ${ }^{3}$, les illustrations

\footnotetext{
N. Charrier-Arrighi $(\bowtie) \cdot$ E. Edy $(\bowtie) \cdot$ E. Fileyssant $(\bowtie)$ Bibliothèque du musée de l'Homme, Muséum national d'histoire naturelle, Direction générale déléguée aux collections, direction des bibliothèques et de la documentation, 17 place du Trocadéro, 75016 Paris, France e-mail : nathalie.charrier-arrighi@mnhn.fr, elise.edy@mnhn.fr, eric.fileyssant@mnhn.fr
}

\footnotetext{
1 bibliotheques.mnhn.fr et http://www.museedelhomme.fr/fr/visitez/ espaces/bibliotheque-recherche-yvonne-oddon

${ }^{2}$ Circulaire n83-219 du 26 mai 1983 du ministère de l'Éducation nationale formalisant la création du réseau des bibliothèques CADIST, centres d'acquisition et de diffusion de l'information scientifique et technique. Les CADIST sont remplacés depuis 2017 par les CollEX, collections d'excellence. Les bibliothèques du Muséum, dont la bibliothèque du musée de l'Homme, sont labellisées CollEx.

${ }^{3}$ Louis Lartet reproduisant un article identique, ou presque, dans plusieurs publications.
}

des ossements et en particulier des crânes sont rares. PrunerBey dans les Annales des sciences naturelles, zoologie, paléontologie de 1868 [6] présente les premières planches hors texte de restes récoltés à Cro-Magnon. Elles sont dessinées par Louveau et seront reprises à l'identique dans les Reliquiae Aquitanicae (1875). Cependant, les gravures de H. Formant, peintre du Muséum ${ }^{4}$, dans Crania ethnica [8], donc publiées sept ans après les Reliquiae et 14 ans après les Annales, sont bien plus connues. Une des explications serait leur parution dans une somme monographique ${ }^{5}$ considérée dès sa publication comme une référence dans le domaine. Elles sont aussi de plus grand format $(35,5 \mathrm{~cm} \times 26 \mathrm{~cm})$, et le dessin de $\mathrm{H}$. Formant est plus précis que celui de Louveau.

\section{Une exposition pour relier la découverte de 1868 à son actualité de 2018}

Dans la vitrine installée à la bibliothèque du musée de l'Homme pour cette exposition «Cro-Magnon : $150^{\mathrm{e}}$ anniversaire d'une découverte (pré)historique ", sont exposés trois éléments de nature différente. Deux ouvrages présentés en alternance, Crania ethnica et Reliquiae Aquitanicae, sont ouverts sur les planches des ossements de Cro-Magnon ${ }^{6}$. Conjointement sont présentées des gravures non reliées, sans doute les premiers tirages remis au dessinateur Formant pour

\footnotetext{
${ }^{4}$ C'est Albert Gaudry qui présente ainsi Formant dans son ouvrage Animaux fossiles et géologie de l'Attique d'après les recherches faites en 1855-1856 et en 1860 sous les auspices de l'Académie des sciences, 1862, F. Savy, Paris, p 6. Le département des Estampes et de la photographie de la Bibliothèque nationale de France possède un portefeuille de gravures de H. Formant. Il s'agit essentiellement de gravures de l'ouvrage susmentionné et celles illustrant Pisciculture et pêche en Chine de Dabry de Thiersant, 1872, Masson, Paris. Aucune illustration de Cro-Magnon ou de paléontologie humaine.

${ }^{5}$ Hypothèse de Noël Coye en réponse à une question posée lors de sa conférence au musée de l'Homme le 14 mai 2018.

${ }^{6}$ Vieillard face et profil, femme face et profil, homme adulte dessus et profil.
} 


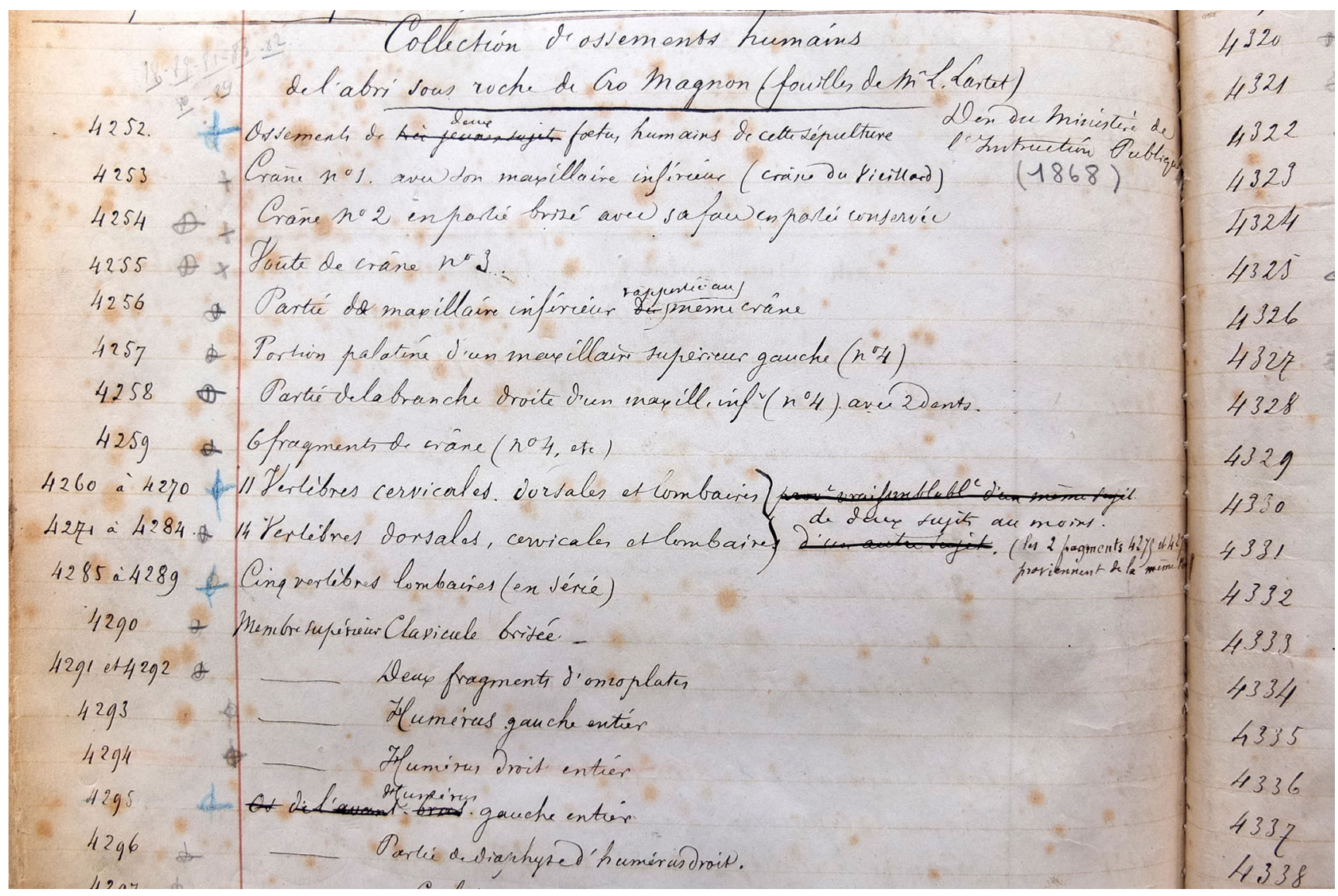

Fig. 1 Détail de la page 302 du registre d'entrée des collections d'anthropologie. Cliché Nathalie Charrier-Arrighi / Highlight of page 302 of the accession register of the anthropological collections. Picture Nathalie Charrier-Arrighi

validation (l'une d'elle est d'ailleurs signée au crayon par Formant). Enfin, pièce la plus originale, le registre d'entrée des collections d'anthropologie ${ }^{7}$ est présenté au public pour la première fois. Il est ouvert à la page 302 (Fig. 1) sur laquelle on peut lire d'une écriture soigneusement calligraphiée : "Collection d'ossements humains de l'abri sous roche de Cro-Magnon (fouilles de M. L. Lartet). Don du ministère de l'Instruction publique [1868 ${ }^{8} »$. Sont listées ensuite sur deux pages les 99 entrées détaillant les différents ossements.

Depuis 150 ans, plusieurs centaines d'articles ont été publiés sur les restes humains trouvés aux Eyzies, témoignant de l'importance scientifique et culturelle de l'événement. Si les scientifiques reviennent périodiquement examiner les découvertes anciennes au gré des avancées techniques et des nouvelles hypothèses, d'autres regards se portent sur les collections.

\footnotetext{
${ }^{7}$ Catalogue des objets renfermés dans la galerie d'anthropologie $d u$ muséum du Jardin des plantes, Paris : 1857-1878, $n^{\circ} 1$ à $n^{\circ} 4999$.

${ }^{8}$ L'année est d'une autre main.
}

\section{Un regard d'artiste sur le crâne de Cro-Magnon}

Quatorze clichés réalisés en 2010 et 2011, dont plusieurs tirés et exposés pour la première fois, sont visibles dans la salle de lecture de la bibliothèque. La photographe Claire Artemyz ${ }^{9}$ se penche sur le crâne du vieillard pour en proposer une lecture d'artiste (Fig. 2). Les variations d'éclairage, de couleur, d'angles de vue et jusqu'aux titres des photographies nous font (re)découvrir os pariétaux, sutures lambdoïdes et sagittales tandis que «La rencontre » met face à face le crâne du vieillard de Cro-Magnon (Homo sapiens) et celui de l'homme de la Chapelle-aux-Saints (Homo neandertha(ensis $)^{10}$.

\footnotetext{
${ }^{9} \mathrm{https}: / / \mathrm{www} \cdot$ artemyzphoto.com/

${ }^{10}$ Un autre tirage de cette photographie est également judicieusement placé dans le parcours permanent de la galerie de l'Homme entre l'atelier de morphing néandertalien et l'alcôve où se trouvent les restes originaux de Cro-Magnon. Il illustre un panneau commémoratif des 150 ans de la découverte.
} 




Fig. 2 Paysages de Cro-Magnon : de trois-quart 1. Photo artistique du crâne de Cro-Magnon exposé dans la salle de lecture de la bibliothèque. Cliché Claire Artemyz / Artistic photo of the Cro-Magnon skull displayed in the reading room of the library. Picturae Claire Artemyz

\section{Valorisation des collections et les nouvelles acquisitions}

Cet anniversaire est aussi l'occasion pour la bibliothèque du musée de l'Homme de valoriser ses collections par un signalement avancé dans les catalogues du Sudoc et du portail de la Direction des bibliothèques et de la documentation du Muséum national d'histoire naturelle ${ }^{11}$. Les deux articles de 1868 de Louis Lartet et de Paul Broca des Bulletins de la Société d'anthropologie de Paris (SAP) y apparaissent désormais. L'intégralité des 180 tomes, fascicules et autres tables des Bulletins de la SAP, des Mémoires de la SAP et des Bulletins et mémoires de la SAP parus entre 1860 et 2018

\footnotetext{
$\overline{11}$ sudoc.abes.fr et bibliotheques.mnhn.fr

12 Beaucoup de fascicules sont malheureusement dans un état trop mauvais, ce qui montre leur forte utilisation, pour pouvoir être empruntés. Mais ils sont désormais disponibles en ligne.
}

sont conservés à la bibliothèque ${ }^{12}$. La totalité des documents scientifiques mentionnés dans la bibliographie du Pôle international de préhistoire des Eyzies ${ }^{13}$ est accessible dans les bibliothèques du Muséum.

La bibliothèque Yvonne-Oddon, au-delà de l'accueil du public, de la valorisation et de la mise à disposition des documents, poursuit toujours une politique d'acquisition d'ouvrages et d'abonnements à des revues. Il s'agit de maintenir son niveau d'excellence et de proposer des collections documentaires en adéquation avec la recherche et l'enseignement en préhistoire et en anthropologie biologique dispensés au musée de l'Homme. Parallèlement, via le portail documentaire de la Direction des bibliothèques, une part importante de la documentation est aujourd'hui proposée en format

\footnotetext{
$\overline{13}$ Pour une bibliographie complète des publications scientifiques relatives à Cro-Magnon, voir sur le site créé par le Pôle international de la préhistoire http://cromagnon150ans.fr/2018/03/12/150-ans-depublications-scientifiques/
} 
électronique. Un chercheur travaillant sur Cro-Magnon y trouvera toute la littérature scientifique, en format papier ou numérique (natif ou numérisé), de 1868 à nos jours. De surcroît, la proximité entre spécimens et documentation facilite le travail de recherche.

\section{Une bibliothèque reflet du musée laboratoire cher à Paul Rivet}

Il n'est pas possible de terminer cette note sans présenter plus généralement la bibliothèque, ses services et ses autres fonds ${ }^{14}$.

Ouverte du lundi au vendredi, de $10 \mathrm{~h}$ à $18 \mathrm{~h}$, cette bibliothèque de recherche de 24 places accueille toute personne intéressée par nos sujets. Un tiers de nos 35000 ouvrages et les trois dernières années de 80 revues sont immédiatement accessibles dans la salle de lecture. Le reste des collections (environ 20000 livres, plus de 700 revues, 30000 tirés à part et 450 estampes) est conservé en magasin mais est consultable dès la demande effectuée. Nous prêtons des ordinateurs portables sur place avec accès gratuit au Wi-Fi, proposons un lecteur agrandisseur et autorisons les photographies pour un usage personnel.

Outre la préhistoire et l'anthropologie biologique, disciplines « historiques » de notre fonds, l'ethnoécologie constitue désormais la troisième thématique phare. En effet, depuis la réouverture en 2015, la bibliothèque Yvonne-Oddon a accueilli les collections de la bibliothèque d'ethnobiologie ${ }^{15}$ et celles du centre de documentation « Ressources des terroirs. Cultures, usages, sociétés $»^{16}$. En 2016 et 2017, des dons d'estampes chinoises et taïwanaises ont ouvert un axe graphique reliant art contemporain, sociétés et représentations animales.

Ces dons issus de collaborations avec des chercheurs et cette exposition construite en synergie avec le musée et les collections naturalistes sont encore, nous l'espérons, le reflet du musée laboratoire cher à Paul Rivet.

Remerciements Que soient remerciés ici Magdalena RuizMarmolejo, conservatrice du patrimoine au musée de l'Homme, qui a attiré notre attention sur le travail de Claire Artemyz, André Delpuech, directeur du Musée, qui a facilité

\section{Informations pratiques}

L'exposition «Cro-Magnon : $150^{\mathrm{e}}$ anniversaire d'une découverte (pré)historique » est présentée jusqu'au 30 novembre 2018 dans la bibliothèque Yvonne-Odon, située au quatrième étage du musée de l'Homme.

À l'occasion des Journées européennes du patrimoine, samedi 15 et dimanche 16 septembre 2018 , les « causeries de la bibliothèque » se penchent cette année sur cette découverte et ses publications historiques. Cette médiation de 30 minutes animée par le personnel de la bibliothèque est ouverte à tout public.

l'installation des photographies, Serge Bahuchet, responsable scientifique des collections d'anthropologie, pour le prêt du catalogue et des gravures et Véronique Laborde, gestionnaire des collections d'anthropologie, pour son aide.

Liens d'intérêts : Les auteurs déclarent ne pas avoir de liens d'intérêts.

\section{Références}

1. Lartet L (1868) Une sépulture des troglodytes du Périgord (crânes des Eyzies). BSAP 3:335-49

2. Broca P (1868) Sur les crânes et ossements des Eyzies. BSAP 3:350-92

3. Lartet L (1868) Sur une sépulture d'anciens troglodytes du Périgord. Rev Soc Savantes 3:279-87

4. Lartet L (1868) Mémoire sur une sépulture des anciens troglodytes du Périgord. Ann Sci Nat Zool Paleontol 10:133-45

5. Pruner-Bey F (1868) Description sommaire de restes humains découverts dans les grottes de Cro-Magnon, près de la station des Eyzies, arrondissement de Sarlat (Dordogne), en avril 1868. Ann Sci Nat Zool Paleontol 10:145-55

6. de Mortillet G (1868) Squelettes humains de l'époque du renne, des Eyzies, Dordogne. Mater Hist Primit Philos Homme 4:150-2

7. Lartet E, Christy H (1875) Reliquiae Aquitanicae: being contributions to the archaeology and palaeontology of Périgord and the adjoining provinces of southern France, Williams \& Norgate, London; JB Baillière, Paris; FA Brockhaus, Leipzic, 2 vol

8. de Quatrefages A, Hamy ET (1882) Crania ethnica : les crânes des races humaines : décrits et figurés d'après les collections du Muséum d'histoire naturelle de Paris, de la Société d'anthropologie de Paris et les principales collections de France et de l'étranger, JB Baillière, Paris, 2 vol.

\footnotetext{
14 http://www.museedelhomme.fr/sites/museedelhomme.fr/files/public/ depliant_bibliotheque.pdf

15 Anciennement rue Cuvier, Direction des bibliothèques et UMR 7206 Écoanthropologie et ethnobiologie.

${ }^{16}$ Centre de documentation "Ressources des terroirs. Cultures, usages, sociétés » (Bourg-en-Bresse), CNRS, Muséum, UMR 7206 Écoanthropologie et ethnobiologie.
} 